\title{
Banach and Edelstein Fixed Point Theorems for Digital Images
}

\author{
Akram Hossain $^{1, *}$, Razina Ferdausi ${ }^{2}$, Samiran Mondal ${ }^{1}$, Harun Rashid ${ }^{1}$ \\ ${ }^{1}$ Department of Mathematics, Jessore University of Science \& Technology, Jessore, Bangladesh \\ ${ }^{2}$ Department of Mathematics, University of Dhaka, Dhaka, Bangladesh \\ *Corresponding author: akrammath90@gmail.com
}

\begin{abstract}
The current paper generalizes the Edelstein fixed point theorem for digital $(\varepsilon, k)$-chainable metric spaces. In order to generalize Edelstein fixed point theorem, we study the digital topological properties of digital images. Further, we establish the Banach fixed point theorem for digital images. We give the notion of digital $(\varepsilon, \lambda, k)$-uniformly locally contraction mapping on digital $(\varepsilon, k)$-chainable metric spaces. Finally, we generalize the Banach fixed point theorem to digital $(\varepsilon, k)$-chainable metric spaces which is known as the Edelstein fixed point theorem for digital images on digital $(\varepsilon, k)$-chainable metric spaces.
\end{abstract}

Keywords: digital image, digital continuity, digital metric space, digital $(\varepsilon, \lambda, k)$-uniformly locally contraction, digital $(\varepsilon, k)$-chainable metric space, Banach fixed point theorem, Edelstein fixed point theorem

Cite This Article: Akram Hossain, Razina Ferdausi, Samiran Mondal, and Harun Rashid, "Banach and Edelstein Fixed Point Theorems for Digital Images.” Journal of Mathematical Sciences and Applications, vol. 5, no. 2 (2017): 36-39. doi: 10.12691/jmsa-5-2-2.

\section{Introduction}

Fixed point theory plays an essential role in various branches of mathematics. The knowledge of the existence of fixed points has relevant applications in many branches of analysis and topology. Besides, it has application to some areas of computer sciences such as computer graphics, image processing, mathematical morphology and so forth. It is very useful to find out a solution if an equation has any solution. Many mathematical problems, originating from various branches of mathematics can be equivalently formulated as fixed point problems meaning that one has to find a fixed point of some functions. In metric spaces, this theory begins with the Banach fixed point theorem (also known as the Banach contraction mapping principle) by Stefan Banach in 1922 [2]. It is an important tool for solution of some problems in mathematics and engineering. Up to now, there are many generalizations of Banach fixed point theorem have been established [12]. In 1961, Michael Edelstein generalized that theorem on $\varepsilon$-chainable metric spaces [10,12].

Digital topology deals with the digital topological properties of $n D, n \in \mathbb{N}$ digital images. It was first introduced by Resenfeld in 1979 [17]. To be specific, he developed the notion of digital continuity for studying $2 D$ and $3 D$ digital images in 1986 [16]. In 1994, Boxer [3,4] expanded the digital versions of several notions such as digital continuous functions including homeomorphisms retractions and homotopies. Few years ago, the concept of digital continuity was extended into the study of $n D$ digital images [13].

The fixed point properties and fixed point theory for digital images were first given by Ege and Karaca [6] named as Lefschetz fixed point theorem. They developed some applications of the Lefschetz fixed point theorem and Nielsen fixed point theorem in digital images to count fixed points [7,9]. They also got some new results associating digital homotopy and fixed point theory [8]. In 2015, they studied the Banach fixed point theorem for digital images [5]. Han [12] refined and improved several notions of that paper such as digital versions of both Cauchy sequence and limit of a sequence in a digital metric spaces. Recently, approximate fixed points and the approximate fixed point property (AFPP) of digitally continuous functions are introduced [4].

This paper is organized as follows. In the first part, we give the required background about the digital images and digital topology. After that, we study the property of the completeness of digital metric spaces. In the next part, we state and prove the Banach fixed point theorem for digital images. Finally, we give the notion of digital $(\varepsilon, k)$ chainable metric spaces and then state and prove the Edelstein fixed point theorem for digital images. Lastly, we give the conclusion.

\section{Preliminaries}

To study the Banach fixed point theorem and Edelstein fixed point theorem from the viewpoint of digital topology, we discuss some basic notions from digital topology:

Let $\mathbb{N}$ and $\mathbb{Z}$ represent the sets of natural numbers and integer numbers respectively. Let $\mathbb{Z}^{n}$, where $n \in \mathbb{N}$ be the sets of lattice points in the $n$-dimensional Euclidean space. It is useful to distinguish between a digital picture and a digital image. We say $\mathbb{Z}^{n}$ is a digital picture [17]. A digital image is a subset of $\mathbb{Z}^{n}$ with the adjacency relation of the 
members of that image.

Definition 1.1: We say that two distinct points $x, y \in \mathbb{Z}^{n}$ are $k$-(or $k(l, n))$ adjacent for $n D$ digital images if they satisfy the followings [13]:

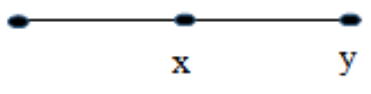

(a) 2-adjacent

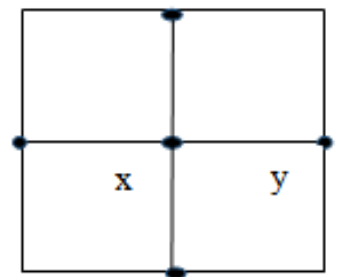

(b) 4-adjacent

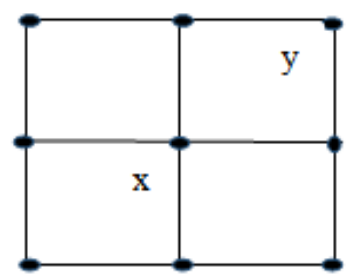

(c) 8-adjacent

Figure 1. Configuration of the digital $k$-connectivity of $\mathbb{Z}^{n}, n \in\{1,2\}[12,17]$

For a natural number $l, 1 \leq l \leq n$, two distinct points

$$
\begin{aligned}
& x=\left(x_{1}, x_{2}, \ldots \ldots, x_{n}\right) \\
& \text { and } y=\left(y_{1}, y_{2}, \ldots . ., y_{n}\right) \in \mathbb{Z}^{n}
\end{aligned}
$$

are $k(l, n)$ or $k$-adjacent if at most $l$ of their co-ordinates differ by \pm 1 and all other points coincide.

Using above fact, we can obtain the $k$-adjacency relations of $\mathbb{Z}^{n}$ as follows [13]:

$$
k:=k(l, n)=\sum_{i=n-l}^{n-1} 2^{n-i} C_{i}^{n},
$$

where $C_{i}^{n}=\frac{n !}{i !(n-i) !}$.

Mathematically, a set $X \subset \mathbb{Z}^{n}$ with the above adjacency relation is called a digital image and denoted by $(X, k)$.

Definition 1.2 [16]: Let $\left(X, k_{0}\right) \subset \mathbb{Z}^{n_{0}}$ and $\left(Y, k_{1}\right) \subset \mathbb{Z}^{n_{1}}$ be digital images. Let $f: X \rightarrow Y$ be a function. Then $f$ is digitally continuous at $x_{0} \in X$ if and only if for every $\varepsilon \geq 1$ there is a $\delta \geq 1$ such that $x \in X$ and $d_{k_{0}}\left(x_{0}, x\right) \leq \delta$ implies $d_{k_{1}}\left(f\left(x_{0}\right), f(x)\right) \leq \varepsilon$.

Definition 1.3 [15]: Let $a, b \in \mathbb{Z}$ with $a \leq b$, then the set

$$
[a, b]_{\mathbb{Z}}=\{z \in \mathbb{Z} \mid a \leq z \leq b\}
$$

with 2-adjacency is called a digital interval.

Using the $k$-adjacency relations of $\mathbb{Z}^{n}$ of (2.1), we define that a digital neighborhood of $x$ in $\mathbb{Z}^{n}$ is the set [16] $N_{k}(x)=\{y \mid y$ is adjacent to $x\}$.

Furthermore, we often use the notation [15]

$$
N_{k}^{*}(x)=N_{k}(x) \cup\{x\} .
$$

Definition 1.4 [11]: A digital image $X \subset \mathbb{Z}^{n}$ is $k$-connected if and only if every pair of different points $x, y \in X$, there is a sequence $\left\{x_{i}\right\}_{i \in[0, l]}$ of points of a digital image $X$ such that $x=x_{0}$ and $y=x_{l}$ with $x_{i}$ and $x_{i+1}$ are $k$-neighbors. Now we can say that there is a simple $k$-path with $l$ elements whose length is the number $l$, denoted by $l_{k}(x, y)$.

For a digital image $(X, k)$ as a generalization of $N_{k}^{*}(x)$, the digital $k$-neighborhood of $x_{0} \in X$ with radius $\varepsilon$ is defined in $X$ to be the following subset [13] of $X$

$$
N_{k}\left(x_{0}, \varepsilon\right)=\left\{x \in X \mid l_{k}\left(x_{0}, x\right) \leq \varepsilon\right\} \cup\left\{x_{0}\right\},
$$

where $l_{k}\left(x_{0}, x\right)$ is the length of a shortest simple $k$-path from $x_{0}$ to $x$ and $\varepsilon \in \mathbb{N}$. Concretely, for $X \subset \mathbb{Z}^{n}$, we obtain

$$
N_{k}(x, 1)=N_{k}^{*}(x) \cap X .
$$

Proposition 2.1 [13]: Let $\left(X, k_{0}\right)$ and $\left(Y, k_{1}\right)$ be digital images in $\mathbb{Z}^{n_{0}}$ and $\mathbb{Z}^{n_{1}}$ respectively. A function $f:\left(X, k_{0}\right) \rightarrow\left(Y, k_{1}\right)$ is digitally $\left(k_{0}, k_{1}\right)$-continuous if and only if for every $x \in X, f\left(N_{k_{0}}(x, 1)\right) \subset N_{k_{1}}(f(x), 1)$.

According to Proposition 2.1, we see that the points $y \in N_{k_{0}}(x, 1)$ is mapped into the points $f(y) \in$ $N_{k_{1}}(f(x), 1)$, which implies that for the points $x, y$ which are $k_{0}$-adjacent a $\left(k_{0}, k_{1}\right)$-continuous map $f$ has the property

$$
\begin{aligned}
& f(x)=f(y) \\
& \text { or, } f(y) \in N_{k_{1}}(f(x)) \cap Y .
\end{aligned}
$$

Definition 2.1 [3,12]: Let $\left(X, k_{0}\right)$ and $\left(Y, k_{1}\right)$ be digital images in $\mathbb{Z}^{n_{0}}$ and $\mathbb{Z}^{n_{1}}$ respectively. Then a map $g: X \rightarrow$ $Y$ is called a $\left(k_{0}, k_{1}\right)$-isomorphism if $g$ is a digital $\left(k_{0}, k_{1}\right)$-continuous, bijective and further $g^{-1}$ is $\left(k_{0}, k_{1}\right)$ continuous which can be denoted by $X \cong_{\left(k_{0}, k_{1}\right)} Y$.

\section{Completeness of the Digital Metric Spaces}

Definition 3.1 [5]: Let $(X, d, k)$ denote a digital metric space with $k$-adjacency relation, where $d$ is the standard Euclidean function on $X \subset \mathbb{Z}^{n}$.

In relation to the study of Banach fixed point theorem and Edelstein fixed point theorem, we recall the following: Definition 3.2 [5]: A sequence $\left\{x_{n}\right\}$ of points of a digital metric space $(X, d, k)$ is a Cauchy sequence if for all $\varepsilon>0$, there exists $N \in \mathbb{N}$ such that for all $n, m>N$, then

$$
d\left(x_{n}, x_{m}\right)<\varepsilon \text {. }
$$

Since the sequence $\left\{x_{n}\right\}$ is defined in the digital metric space $(X, d, k)$, Han [12] observed that the Euclidean distance between any two distinct points $x, y \in X$ is greater than or equal to 1 as follows:

Proposition 3.1 [12]: In a digital metric space $(X, d, k)$, consider two points $x_{i}, x_{j}$ in a sequence $\left\{x_{n}\right\}$ of $X$ such that they are $k$-adjacent i.e. $x_{i} \in N_{k}\left(x_{j}, 1\right)$ or $x_{j} \in$ $N_{k}\left(x_{i}, 1\right)$, and $x_{i} \neq x_{j}$. Then they have the Euclidean distance $d\left(x_{i}, x_{j}\right) \in\left\{\sqrt{t} \mid t \in[1, l]_{\mathbb{Z}}\right\}$. 
Proof: Since the Definition 2.1 implies that any two distinct points $x_{i}, x_{j} \in\left\{x_{n}\right\}$ in the digital metric space $(X, d, k)$ has at most $l$ of their co-ordinates which differs by \pm 1 and all other coincide. Thus the Euclidean distance $d\left(x_{i}, x_{j}\right) \in\{1, \sqrt{2}, \cdots \cdots, \sqrt{t}\}$ depending on the position of that two points. For instance, in Figure 1 (a), the Euclidean distance of any two points 2 -adjacent in $\mathbb{Z}$ is 1 . In Figure 1 (b), the Euclidean distance of any two points 4adjacent in $\mathbb{Z}^{2}$ is 1 . In Figure 1 (c), the Euclidean distance of any two points 8 -adjacent in $\mathbb{Z}^{2}$ is either 1 or $\sqrt{2}$ depending on the position of the given two points. For instance, consider two 8-adjacent points of $p(0,0)$ in $\mathbb{Z}^{2}$ are $p_{1}(0,1)$ and $p_{2}(1,1)$. Now the Euclidean distance of these two points are $d\left(p, p_{1}\right)=1$ and $d\left(p, p_{2}\right)=\sqrt{2}$. Similarly, in three dimensional case, we have Euclidean distances $\left\{\sqrt{t} \mid t \in[1,3]_{\mathbb{Z}}\right\}$ depending on the positions of the two points.

Thus by using the above Proposition 3.1, we obtain the following:

Proposition 3.2 [12]: A sequence $\left\{x_{n}\right\}$ of points of a digital metric space $(X, d, k)$ is a Cauchy sequence if and only if there is $N \in \mathbb{N}$ such that for all $n, m>N$, we have

$$
d\left(x_{n}, x_{m}\right)<1
$$

i.e. $x_{n}=x_{m}$ for all $n, m>N$.

By using the Proposition 3.1, we can define the convergency of a sequence of a digital metric space $(X, d, k)$ as follows:

Definition 3.2 [12]: A sequence $\left\{x_{n}\right\}$ of points of a digital metric space $(X, d, k)$ converges to a limit $L \in X$ if there is $N \in \mathbb{N}$ such that for all $n>N$, we have

$$
x_{n}=L \text {, i.e. } x_{n}=x_{n+1}=x_{n+2}=\cdots \cdots=L .
$$

Definition 3.3 [12]: A digital metric space $(X, d, k)$ is a complete digital metric space if any Cauchy sequence $\left\{x_{n}\right\}$ of points of $(X, d, k)$ converges to a point $L \in X$.

\section{Banach Fixed Point Theorem for Digital Contraction Mapping}

Definition 4.1 [5]: Let $(X, d, k)$ be a digital metric space and $f:(X, d, k) \rightarrow(X, d, k)$ be a self-mapping. If there exists $\lambda \in[0,1)$ such that for all $x, y \in X$, we have $d(f(x), f(y)) \leq \lambda d(x, y)$ then $f$ is called a digital contraction mapping.

Proposition 4.1: Every digital contraction mapping is digitally continuous.

Proof: Let $(X, d, k)$ be a digital metric space and $f:(X, d, k) \rightarrow(X, d, k)$ be a digital contraction mapping. Let $a, b \in X$ and $d(a, b)=1$ by the Proposition 3.1, we have

$$
\begin{gathered}
d(f(a), f(b)) \leq \lambda d(a, b)=\lambda .1=\lambda \\
\text { i.e. } d(f(a), f(b))<\lambda,
\end{gathered}
$$

where $\lambda \in[0,1)$ for all $a, b \in X$. Then $f$ is a digitally $(k, k)$-continuous.

Motivated by the paper [5], here we give the Banach fixed point theorem in the following way:
Theorem 4.1 (Banach contraction principle for digital images): Let $(X, d, k)$ be a complete digital metric space with Euclidean metric $d$ on $X \subset \mathbb{Z}^{n}$. Let $f:(X, d, k) \rightarrow$ $(X, d, k)$ be a digital contraction mapping. Then $f$ has a unique fixed point.

Proof: For notational purposes, we define $f^{n}(x), x \in$ $X$ and $n \in \mathbb{N}$, inductively by $f^{0}(x)=x$ and $f^{n+1}(x)=$ $f\left(f^{n}(x)\right)$. To show existence, we select $x \in X$. We first show that $\left\{f^{n}(x)\right\}$ is a Cauchy sequence.

Notice for $n \in \mathbb{N}$ that

$$
\begin{aligned}
& d\left(f^{n}(x), f^{n+1}(x)\right) \leq \lambda d\left(f^{n-1}(x), f^{n}(x)\right) \\
& \leq \cdots \cdots \leq \lambda^{n} d(x, f(x)) .
\end{aligned}
$$

Thus for $m>n$, we have

$$
\begin{aligned}
d & \left.d f^{n}(x), f^{m}(x)\right) \\
\leq & d\left(f^{n}(x), f^{n+1}(x)\right)+d\left(f^{n+1}(x), f^{n+2}(x)\right) \\
& +\cdots \cdots+d\left(f^{m-1}(x), f^{m}(x)\right) \\
= & \lambda^{n}\left[1+\lambda+\cdots \cdots+\lambda^{m-n-1}\right] d(x, f(x)) \\
= & \lambda^{n} \sum_{s=0}^{m-n-1} \lambda^{s} d(x, f(x)) \\
\leq & \lambda^{n} \sum_{s=0}^{\infty} \lambda^{s} d(x, f(x)) \\
\therefore & d\left(f^{n}(x), f^{m}(x)\right) \leq \frac{\lambda^{n}}{1-\lambda} d(x, f(x))<1 .
\end{aligned}
$$

By Proposition 3.1, we have $d\left(f^{n}(x), f^{m}(x)\right)=0$, which implies $f^{n}(x)=f^{m}(x)$. This shows that $\left\{f^{n}(x)\right\}$ is a Cauchy sequence and since $X$ is complete, so there exists $p \in X$ such that $\lim _{n \rightarrow \infty} f^{n}(x)=p$.

Moreover, the continuity of $f$ yields

$$
\begin{aligned}
& p=\lim _{n \rightarrow \infty} f^{n+1}(x)=\lim _{n \rightarrow \infty} f\left(f^{n}(x)\right) \\
& =f\left(\lim _{n \rightarrow \infty} f^{n}(x)\right)=f(p) .
\end{aligned}
$$

Therefore, $p$ is a fixed point of $f$.

For uniqueness, suppose that $q$ is a another fixed point of $f$ such that $f(q)=q$ and $p \neq q$, then

$$
\begin{aligned}
& d(p, q)=d(f(p), f(q)) \leq \lambda d(p, q) \\
& \Rightarrow(1-\lambda) d(p, q) \leq 0 .
\end{aligned}
$$

But $1-\lambda>0$, so $d(p, q)=0$. Therefore, $p=q$ and our proof is completed.

\section{Edelstein Fixed Point Theorem for Digital $(\varepsilon, k)$ Chainable Metric Spaces}

An extension of Banach fixed point theorem was given by Edelstein to a class of mappings on $\varepsilon$ - chainable metric spaces [10]. Based on that we discuss Edelstein fixed point theorem for digital $(\varepsilon, k)$ chainable metric spaces 
from the viewpoint of digital topology. In this regard, we discuss digital $\varepsilon$-chain, $(\varepsilon, \lambda, k)$-uniformly locally contractive mapping and $\varepsilon$-chainable metric spaces for digital topology.

Definition 5.1: Let $\left\{x_{n}\right\}$ be a sequence of points of a digital metric space $(X, d, k)$ and suppose $\varepsilon=1$. Then the sequence $\left\{x_{n}\right\}$ is said to be $(\varepsilon, k)$ chain joining $x_{0}$ and $x_{n}$ if $x_{i-1}$ and $x_{i}$ are neighbors, $i \in\{1,2, \cdots \cdots, n\}$, i.e., $d\left(x_{i-1}, x_{i}\right) \leq 1$.

Definition 5.2: The digital metric space $(X, d, k)$ is said to be digitally $(\varepsilon, k)$-chainable metric space if each pair $(x, y)$ of its points, there exists an $\varepsilon$-chain joining $x$ and $y$. Definition 5.3: A mapping $f:(X, d, k) \rightarrow(X, d, k)$ is called digitally $(\varepsilon, \lambda, k)$-uniformly locally contractive if there exists $\epsilon=1$ and $\lambda \in[0,1)$ such that $d(x, y) \leq 1$ which implies $d(f(x), f(y)) \leq \lambda d(x, y)$ for each $x, y \in X$.

Theorem 5.1 (Edelstein fixed point theorem for digital images): Let $(X, d, k)$ be a complete digital $(\varepsilon, k)$ chainable metric space and $f:(X, d, k) \rightarrow(X, d, k)$ be a digital $(\varepsilon, \lambda, k)$ - uniformly locally contractive mapping. Then $f$ has a unique fixed point $c$ in $X$.

Proof: Given $(X, d, k)$ is a complete digital $(\varepsilon, k)$ chainable metric space. Now we define a new metric by

$$
d_{\mathcal{E}}(x, y)=\inf \sum d\left(x_{i-1}, x_{i}\right) \text { for } x, y \in X,
$$

where infimum is taken over all $(\varepsilon, k)$ chains $x_{0}, x_{1}, \cdots \cdots, x_{n}$ joining $x_{0}=x$ and $x_{n}=y$. Then the metric $d_{\varepsilon}$ on $X$ satisfies

(i) $d(x, y) \leq d_{\varepsilon}(x, y)$

(ii) $d(x, y)=d_{\varepsilon}(x, y)$ for $d(x, y)<1$.

Then the metric $\left(X, d_{\varepsilon}, k\right)$ is complete whenever $(X, d, k)$ is complete. With given $x, y \in X$ and any $(\varepsilon, k)$-chains $x_{0}, x_{1}, \cdots \cdots, x_{n}$ joining $x_{0}=x$ and $x_{n}=y$, we have

$$
d(x, y)=\sum_{i=1}^{n} d\left(x_{i-1}, x_{i}\right),
$$

where $d\left(x_{i-1}, x_{i}\right) \leq 1$ for $i=1,2, \cdots \cdots, n$.

Now we can write

$$
d(f(x), f(y)) \leq \sum_{i=1}^{n} d\left(f\left(x_{i-1}\right), f\left(x_{i}\right)\right) .
$$

Hence, by the Definition 5.3, we have

$$
\sum_{i=1}^{n} d\left(f\left(x_{i-1}\right), f\left(x_{i}\right)\right) \leq \lambda \sum_{i=1}^{n} d\left(x_{i-1}, x_{i}\right) \leq \lambda n<1 .
$$

So $f\left(x_{0}\right), f\left(x_{1}\right), \cdots \cdots, f\left(x_{n}\right)$ is an $(\varepsilon, k)$-chain joining $f(x)$ and $f(y)$.

Then

$$
\begin{aligned}
d_{\varepsilon}(f(x), f(y)) & \leq \sum_{i=1}^{n} d\left(f\left(x_{i-1}\right), f\left(x_{i}\right)\right) \\
& \leq \lambda \sum_{i=1}^{n} d\left(x_{i-1}, x_{i}\right) .
\end{aligned}
$$
have

$$
d_{\varepsilon}(f(x), f(y)) \leq \lambda d_{\varepsilon}(x, y) .
$$

Since $f$ has a unique fixed point $c \in X$, given by $\lim _{n \rightarrow \infty} d_{\varepsilon}\left(f^{n}(x), c\right)=0$ for any $x \in X$ [By Banach fixed point theorem].

But by $(i)$, we have $\lim _{n \rightarrow \infty} d\left(f^{n}(x), c\right)=0$. Therefore, our proof is completed.

\section{Conclusion}

We have reviewed some notions of digital topology for image processing technique. Further, we have studied the Banach fixed point theorem for digital images. At last, we have discussed the digital version of Edelstein fixed point theorem for digital images which is the generalization of Banach fixed point theorem in metric spaces. We hope that all results in this paper help us to understand better structure of digital images.

In future, we will study the other fixed point theorem for digital images in digital topology.

\section{Conflicts of Interest}

The authors declare that there is no conflicts of interest regarding the publication of this paper.

\section{References}

[1] R. P. Agarwall, M. Meehan, D. O’Regan, Fixed Point Theory and Applications, Cambridge University Press, 2001.

[2] S. Banach, Sur less opérations dans les ensembles abstraits et leur applications aux equations integrates, Fund. Math. 3(1922), 133-181.

[3] L. Boxer, Digitally Continuous Function, Pattern Recognition Letter, 15(1994), 833-839.

[4] L. Boxer, O. Ege, I. Karaca, J. Lopez and J. Louwsma, Digital fixed points, approximate fixed points, and universal functions, Applied General Topology, 17(2), 159-172 (2016).

[5] O. Ege, I. Karaca, Banach fixed point theorem for digital images, J. Nonlinear. Sci. Appl., 8(2015), 237-245.

[6] O. Ege, I. Karaca, Lefschetz fixed point theorem for digital images, Fixed Point Theory and Applications, 2013, 2013:253.

[7] O. Ege and I. Karaca, Applications of the Lefschetz number to digital images, Bulletin of the Belgian Mathematical Society Simon Stevin, 21(5), 823-839 (2014).

[8] O. Ege and I. Karaca, Digital homotopy fixed point theory, Comptes Rendus Mathematique, 353(11), 1029-1033 (2015).

[9] O. Ege and I. Karaca, Nielsen fixed point theory for digital images, Journal of Computational Analysis and Applications, 22(5), 874-880 (2017).

[10] M. Edelstein, An Extention of Banach's Contraction Principle, Amer. Math. Soc., 12(1961), 7-10.

[11] G.T. Herman, Oriented surfaces in digital spaces, CVGIP: Graphical Models and Image Processing, 55(1993).

[12] S. E. Han, Banach fixed point theorem from the viewpoint of digital topology, J. Nonlinear. Sci. Appl. 9(2016), 895-905.

[13] S. E. Han, Non-product property of the digital fundamental group, Inform. Sci., 171(2005), 73-91.

[14] M. C. Joshi, R.K. Bose, Some Topics in Non-linear Functional Analysis, Wiley Eastern Limited, New Delhi 110 002, 1985.

[15] T. Y. Kong, A. Rosenfeld, Topological Algorithms for the Digital Image Processing, Elsevier Science, Amsterdam, 1996.

[16] A. Rosenfeld, Continuous functions on digital pictures, Pattern Recognition Letter, 4(1986), 177-184.

[17] A. Rosenfeld, Digital topology, Amer. Math. Soc., 86(1979), 621-630. 